\title{
Hypericum extract versus imipramine or placebo in patients with moderate depression: randomised multicentre study of treatment for eight weeks
}

\author{
Michael Philipp, Ralf Kohnen, Karl-O Hiller
}

Bezirkskrankenhaus Landshut, D-84034 Landshut, Germany Michael Philipp professor of psychiatry

Imerem Institute for Medical Research Management and Biometrics, D-90478

Nuremberg, Germany

Ralf Kohnen professor of psychology

Steiner

Arzneimittel, D-12207 Berlin,

Germany

Karl-O Hiller senior research fellow

Correspondence to: K-O Hiller

kohiller.steiner@ -online.de

BMJ 1999;319:1534-9

website

extra

The sample size calculation and a chart showing the flow of participants through the trial appear on the BMJ's website

www.bmj.com

\section{Abstract}

Objectives To assess the efficacy and safety of hypericum extract (STEI 300, Steiner Arzneimittel, Berlin) compared with imipramine and placebo in patients in primary care with a current episode of moderate depression.

Design Randomised, double blind, multicentre, parallel group trial for 8 weeks.

Setting Trained panel of 18 general practitioners from four German states: Bavaria, Berlin, Rhineland Palatinate, and Saxony.

Participants 263 patients (66 men, 197 women) with moderate depression according to ICD-10

(international classification of diseases, 10th revision) codes F32.1 and F33.1.

Interventions $1050 \mathrm{mg}$ hypericum extract $(350 \mathrm{mg}$ three times daily), $100 \mathrm{mg}$ imipramine (50 mg, $25 \mathrm{mg}$, and $25 \mathrm{mg}$ daily), or placebo three times daily.

Main outcome measures Change from baseline score on the 17 item version of the Hamilton depression scale, the Hamilton anxiety scale, the clinical global impressions scale, Zung's self rating depression scale, and SF-36, and adverse events profile.

Results Hypericum extract was more effective at reducing Hamilton depression scores than placebo and as effective as imipramine (mean - 15.4 (SD 8.1), - 12.1 (7.4), and -14.2 (7.3) respectively). Comparable results were found for Hamilton anxiety and clinical global impressions scales and were most pronounced for the Zung self rating depression scale. Quality of life was more improved in the standardised mental component scale of the SF-36 with both active treatments than with placebo but in the physical component scale was improved only by hypericum extract compared with placebo. The rate of adverse events with hypericum extract was in the range of the placebo group but lower than that of the imipramine group $(0.5,0.6$, and 1.2 events per patient respectively)

Conclusions At an average dose of $350 \mathrm{mg}$ three times daily hypericum extract was more effective than placebo and at least as effective as $100 \mathrm{mg}$ imipramine daily in the treatment of moderate depression. Treatment with hypericum extract is safe and improves quality of life.

\section{Introduction}

Depressive disorders are recognised as disabling psychiatric illnesses, with lifetime prevalences of over $10 \%$ in the general population. ${ }^{1}$ These conditions are underdiagnosed and frequently undertreated. ${ }^{2}$ The prevalence of depressive disorders in primary care in Germany has been estimated at $8.6 \%{ }^{3}$

A systematic review of 27 clinical studies of different hypericum preparations concluded that they are more effective than placebo in the treatment of mild to moderate depression. ${ }^{4}$ Previous research has, however, been criticised for methodological shortcomings particularly in equivalence trials with other antidepressants. ${ }^{56}$ Most comparative trials concluded that hypericum products were as effective as synthetic antidepressants, but the trials lacked a placebo group. ${ }^{4} \mathrm{We}$ report the results of the first three arm study in which hypericum extract (STEI 300, Steiner Arzneimittel, Berlin) was compared with a tricyclic antidepressant and a placebo in the treatment of patients for eight weeks with moderate depression. We followed recent statistical requirements and recommendations. ${ }^{7}$

\section{Methods}

Our study was conducted according to the declaration of Helsinki and the European guidelines for good clinical practice. ${ }^{8}$ The study protocol was approved by four medical ethics committees in Germany.

\section{Design, efficacy, and safety evaluation}

We conducted a double blind, randomised, placebo controlled, multicentre trial for eight weeks. Patients were screened for one week before treatment, which included a washout period for previous antidepressants. Efficacy and safety were evaluated after 1, 2, 4, 6, and 8 weeks with the 17 item version of the Hamilton depression rating scale, the Hamilton anxiety scale, the clinical global impressions scale, and the Zung self rating depression scale. Data for evaluation of safety comprised adverse events, clinically relevant changes in ECG, measurements of vital signs, and physical examinations. Quality of life was rated by the patients with SF-36. All participant doctors were trained by the principal investigator (MP) to improve reliability in the rating of the main outcome criterion, the Hamilton depression total score. Blocks of 12 patients were randomised in the ratio 5:5:2 for hypericum extract STEI 300 , imipramine, and placebo respectively.

\section{Patients}

Patients were continuously selected from the cohorts of depressed patients by a panel of 18 general practitioners. Box 1 summarises the main inclusion and exclusion criteria.

\section{Drugs}

During the screening period patients remained untreated until a baseline evaluation of efficacy and safety was completed. All patients were treated with three capsules of trial drug daily. Hypericum extract STEI 300 (extracted from St John's wort with 60\% ethanol w/w; composition of pharmaceutically relevant substances $0.2 \%-0.3 \%$ hypericin and pseudohypericin and 2\%-3\% hyperforin according to high 


\section{Box 1: Main inclusion and exclusion criteria}

Inclusion

- Men and women aged 18-65

- Diagnosis of a moderate depressive episode according to ICD-10 (international classification of diseases, 10th revision) codes F32.1 and F33.1

- Minimum total score of 18 on the 17 item version of the Hamilton depression rating scale

- A clinical global impressions rating of severity (item 1) of moderately, markedly, or severely ill

- Depression duration a minimum of four weeks and a maximum of two years

Exclusion

- Mild and severe depressive disorders according to ICD-10 codes F32.0, F33.0, F32.2, F33.2, F32.3, and F33.3

- Bipolar disorders according to ICD-10 codes F 31.x

- Comorbidity from alcohol or drug dependence according to ICD-10 codes F10-F19

- Suicidal risk (assessed by item 10 of the Montgomery Asberg depression rating scale)

- Long term prophylaxis with lithium or carbamazepine

- Non-sufficient washout phase of previous psychotropic drug

- Any interfering psychotropic drug taken concurrently

- Any previous long term ( $>3$ months) treatment with benzodiazepines

- Patients at general and specific risk (imipramine contraindications)

performance liquid chromatography) was given in a constant dose of $350 \mathrm{mg}$ per capsule (total daily dose $1050 \mathrm{mg}$ ). Imipramine was titrated in a dose of $50 \mathrm{mg}$ on the first treatment day, $75 \mathrm{mg}$ on days $2-4$, and 100 $\mathrm{mg}(50 \mathrm{mg}, 25 \mathrm{mg}$, and $25 \mathrm{mg}$ ) thereafter. The capsules were identical in appearance and taste and were packed in identical containers identified only by patient number. Compliance with drug intake (80\%-120\% of the prescribed dose per protocol) was checked by counting pills during and at the end of the treatment period.

\section{Statistical analysis}

Changes in scores on the Hamilton depression rating scale between baseline and final assessment of efficacy were defined in the study protocol as primary efficacy criterion. The definitions of endpoints for confirmative testing (six weeks for comparison with placebo, eight weeks for comparison with imipramine) are based on the recommendations for evaluating antidepressive efficacy. ${ }^{9}$ Three hypotheses were tested in a hierarchical procedure: superiority of hypericum extract over placebo, equivalence between hypericum extract and imipramine, and superiority of active control over placebo. Confirmatory hypothesis testing was to be stopped if the first or second null hypothesis could not be rejected ( $\mathrm{P} \leqslant 0.025$, one sided test problem). Multiple two sample $t$ tests were used for statistical hypothesis testing. Two sided 95\% confidence intervals, including centres as stratum, ${ }^{10}$ were calculated for comparisons between the treatments in this trial.
All other scales were analysed as secondary efficacy criteria with two sample tests ( $t$ test, $\chi^{2}$ test) and interpreted on an exploratory basis. The evaluation of efficacy was based on the intention to treat analysis, using the method of last observation carried forward in the case of patients who prematurely dropped out of the study. Safety analysis included all patients who received at least one dose of any test drug and had safety assessments after baseline. The qualification of patients for the different analyses populations was defined in a blind review evaluation before unblinding the random code. Statistical analyses were performed with SAS version 6.12 .

\section{Results}

\section{Patients}

Overall, 263 patients were randomised in 18 centres (median 12 patients, 3 to 24 per centre) from October 1996 to April 1998 (see website). In the intention to treat population of 251 patients-hypericum extract (100 patients), imipramine (105), and placebo (46) - the three treatment groups were comparable for sex, age, height, weight, diagnoses according to the ICD-10, and scores on the initial Hamilton depression and Hamilton anxiety scales (table 1).

\section{Compliance and efficicay}

Overall compliance was satisfactory-on average patients took a mean $100 \%(4 \%)$ of their pills (range 69\%-108\%). Figure 1 shows the increasingly larger improvements at any assessment after baseline (with initial Hamilton depression total scores of 22 and 23 points on average) for hypericum extract and imipramine compared with placebo, with the largest improvement with hypericum extract. The placebo group also showed considerable improvements during the study.

The primary endpoint analysis showed that hypericum extract was more effective than placebo after six weeks of treatment (change from baseline $-3.1,-5.4$

Table 1 Characteristics of patients with moderate depressive episodes (safety population $\mathrm{n}=263$ ) and baseline values in efficacy criteria (intention to treat population $\mathrm{n}=251$ ). Values are means (SDs) unless stated otherwise

\begin{tabular}{|c|c|c|c|c|}
\hline \multirow[b]{2}{*}{ Characteristics } & \multirow[b]{2}{*}{$\begin{array}{l}\text { All patients } \\
(n=263)\end{array}$} & \multicolumn{3}{|c|}{ Group } \\
\hline & & $\begin{array}{l}\text { Placebo } \\
(n=47)\end{array}$ & $\begin{array}{l}\text { Hypericum extract } \\
(\mathrm{n}=106)\end{array}$ & $\begin{array}{c}\text { Imipramine } \\
(\mathrm{n}=110)\end{array}$ \\
\hline No $(\%)$ of men & $66(25)$ & $9(19)$ & $26(25)$ & $31(28)$ \\
\hline No $(\%)$ of women & $197(75)$ & $38(81)$ & $80(76)$ & $79(72)$ \\
\hline Mean age (years) & $47(12)$ & $43(12)$ & $47(12)$ & $48(12)$ \\
\hline \multicolumn{5}{|c|}{ No (\%) with diagnosis of moderate depressive episode*: } \\
\hline F32.1: first manifestation & $203(77)$ & $38(81)$ & $84(79.2)$ & $81(74)$ \\
\hline F33.1: recurrent episode & $60(23)$ & $9(19)$ & $22(21)$ & $29(26)$ \\
\hline \multicolumn{5}{|l|}{ Disease history (months): } \\
\hline Depressive disorder & $7.8(7.8)$ & $7.7(7.6)$ & $7.2(6.9)$ & $8.5(8.6)$ \\
\hline Current episode & $4.5(4.8)$ & $4.7(5.5)$ & $4.3(4.3)$ & $4.6(4.9)$ \\
\hline \multicolumn{5}{|l|}{ Baseline valuest: } \\
\hline Hamilton depression scale & $22.6(4.1)$ & $22.7(4.0)$ & $22.7(4.2)$ & $22.2(4.2)$ \\
\hline Hamilton anxiety scale & $22.0(6.4)$ & $21.4(7.4)$ & $22.0(6.1)$ & $22.4(6.2)$ \\
\hline Zung self rating depression scale & $55.3(8.7)$ & $55.3(7.5)$ & $55.1(9.2)$ & $55.5(8.8)$ \\
\hline \multicolumn{5}{|c|}{ No (\%) clinical global impression (item 1): } \\
\hline Moderately ill & $91(36)$ & $17(37)$ & 39 (39) & $35(33)$ \\
\hline Markedly ill & $151(60)$ & $26(57)$ & $60(60)$ & $65(62)$ \\
\hline Severely ill & $9(4)$ & $3(7)$ & $1(1)$ & $5(5)$ \\
\hline
\end{tabular}

${ }^{*}$ According to international classification of diseases, 10th revision.

†Patient numbers of intention to treat population (all patients $n=251$, placebo group $n=46$, hypericum extract group $n=100$, and imipramine group $n=105$ ) 


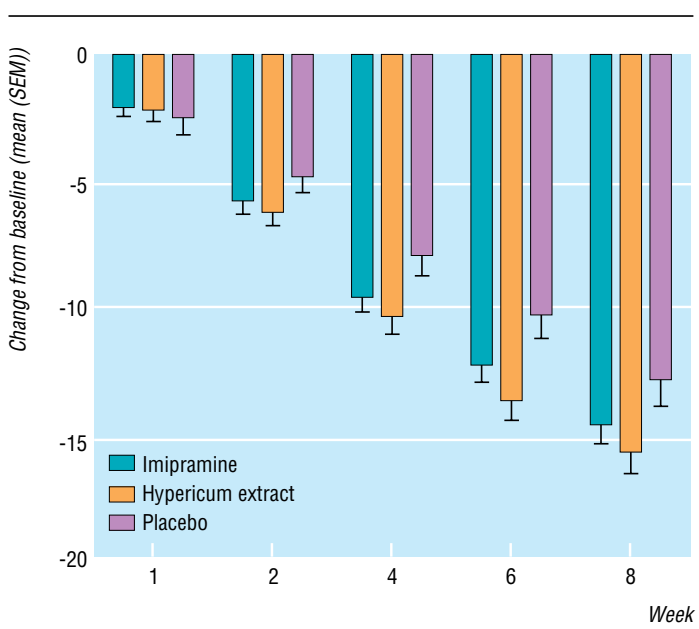

Fig 1 Mean (SEM) change from baseline in Hamilton depression total scores at all visits in intention to treat population. Observed cases analysis at weeks 1,2 , and 4 , and last observation carried forward analysis for weeks 6 and 8

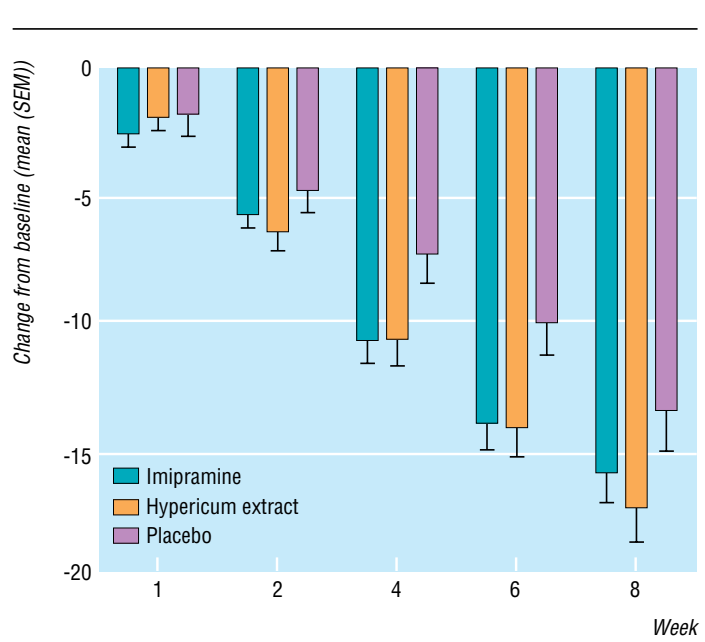

Fig 2 Mean (SEM) change from baseline in Zung self rating depression total score at all visits in intention to treat population. Observed cases analysis for weeks 1, 2, and 4, and last observation carried forward analysis for weeks 6 and 8

to -1.5 , negative values indicate better efficacy of hypericum extract; table 2). In the second hypothesis test, equivalent efficacy was shown between hypericum extract and imipramine $(-1.2,95 \%$ confidence interval -2.6 to 0.6 ). For imipramine we found only a strong tendency towards it being more effective than placebo after six weeks of treatment $(-1.7,-3.89$ to -0.05$)$. However, the $95 \%$ confidence interval did not include the zero point of equal efficacy. The differences between the two treatment groups and placebo were comparable at week 8 , but slightly less pronounced than in week 6 owing to further improvement in the placebo group.

In the Hamilton anxiety scale, improvements were greater after six weeks of treatment with hypericum extract than with placebo $(-2.7,-5.44$ to -0.51$)$ and less pronounced in the imipramine group $(-1.8$, -4.43 to 0.46$)$. Compared with placebo $(50 \%)$, the number of patients who improved in the clinical global impressions scale was noticeably higher under active treatments: $74 \%$ in the hypericum group (24\%, $13 \%$ to $39 \%), 71 \%$ in the imipramine group (21\%, $9 \%$ to $35 \%$ ). Differences between the active treatment groups and the placebo group were smaller at week 8 (table 2).

In the Zung self rating depression scale both treatments were superior to placebo at week 6 and hypericum extract was also more efficacious at week 8, showing a higher sensitivity for active control versus placebo (fig 2, table 2).

\section{Quality of life}

The standardised component scales of the SF-36 are reported in this paper as a summary information on quality of life. The differences between both drugs compared with placebo were larger in the mental component scale than in the physical component scale. In both scales hypericum extract was more effective than placebo (mental component 5.3, 1.9 to 8.7, physical component $3.5,1.1$ to 6.1 ); a positive value indicates better efficacy of hypericum extract (table 2).

\section{Safety}

In the safety population, $22 \%$ ( 0.5 events per patient) of the hypericum group and $46 \%$ (1.2) of the imipramine group reported adverse events with treatment (table 3); the placebo group (19\%) was comparable to that of the hypericum group (0.6). Overall, the most frequent adverse event was dry mouth $(38 \%$ in the imipramine group). The most frequently reported adverse event in the hypericum group was nausea $(8 \%, 3.3 \%$ to $14.3 \%)$. No serious adverse events were reported in the hypericum and imipramine groups. One patient in the placebo group attempted suicide but did eventually complete the study although with little progress.

\section{Discussion}

\section{Efficacy of hypericum extract}

Our results showed that hypericum extract STEI 300 was more effective than placebo after six weeks (confirmatory testing) and eight weeks (exploratory testing) of treatment in primary care patients with moderate depression. Our study sample was representive of some confounding demographic variables and disease characteristics in patients in primary care with depressive disorders, for example, age and sex distribution, severity, chronicity. According to Montgomery, a difference of more than three points in change from baseline on the Hamilton depression scale between both treatments is not only statistically but also clinically relevant. ${ }^{11}$ Following current recommendations for equivalence trials, ${ }^{12}$ we showed that hypericum extract was equivalent in efficacy to imipramine after eight weeks of treatment.

\section{Efficacy of imipramine and placebo effects}

Although the improvement in the imipramine group was considerable $(63 \%$ and $67 \%$ on the Hamilton depression scale at weeks 6 and 8), indicating effective responder rates for antidepressive treatment, only a strong tendency towards superior efficacy over placebo could be found in the Hamilton depression scale. In our trial the dosage of $100 \mathrm{mg}$ imipramine 
Table 2 Changes from baseline in intention to treat population for evaluation of efficacy. Values are means (SDs) unless stated otherwise

\begin{tabular}{|c|c|c|c|c|c|c|}
\hline \multirow[b]{2}{*}{ Variable (visit) } & \multicolumn{3}{|c|}{ Group } & \multicolumn{3}{|c|}{ Difference $(95 \% \mathrm{Cl})^{*}$} \\
\hline & Placebo & $\begin{array}{c}\text { Hypericum } \\
\text { extract }\end{array}$ & Imipramine & $\begin{array}{c}\text { Hypericum } \\
\text { extract-placebo }\end{array}$ & Imipramine-placebo & $\begin{array}{c}\text { Hypericum } \\
\text { extract-imipramine }\end{array}$ \\
\hline \multicolumn{7}{|c|}{ Hamilton depression rating } \\
\hline \multicolumn{7}{|l|}{ Score: } \\
\hline Week 6 & $-10.3(6.1)$ & $-13.4(7.8)$ & $-12.0(6.6)$ & $-3.1(-5.44$ to -1.46$)$ & $-1.7(-3.89$ to -0.05$)$ & $-1.4(-2.73$ to 0.31$)$ \\
\hline Week 8 & $-12.1(7.4)$ & $-15.4(8.1)$ & $-14.2(7.3)$ & $-3.3(-5.60$ to -1.33$)$ & $-2.1(-4.37$ to -0.04$)$ & $-1.2(-2.57$ to 0.60$)$ \\
\hline \multicolumn{7}{|l|}{ Responder (\%)†: } \\
\hline Week 6 & 47.8 & 67.0 & 62.9 & 19.2 (7.4 to 35.7$)$ & $15.1(2.4$ to 31.0$)$ & $4.1(-8.3$ to 15.3$)$ \\
\hline Week 8 & 63.0 & 76.0 & 66.7 & $13(1.7$ to 27.2$)$ & $3.7(-8.9$ to 17.7$)$ & $9.3(-2.0$ to 20.0$)$ \\
\hline \multicolumn{7}{|c|}{ Total score without item 11: } \\
\hline Week 6 & $-9.7(6.2)$ & $-13.4(7.8)$ & $-12.5(7.0)$ & $-3.7(-5.33$ to -1.67$)$ & $-2.8(-3.77$ to -0.24$)$ & $-0.9(-2.64$ to 0.19$)$ \\
\hline Week 8 & $-11.9(7.6)$ & $-15.3(8.3)$ & $-14.7(8.0)$ & $-3.4(-5.52$ to -1.60$)$ & $-2.8(-4.31$ to -0.30$)$ & $-0.6(-2.47$ to 0.49$)$ \\
\hline \multicolumn{7}{|c|}{ Hamilton anxiety scale } \\
\hline \multicolumn{7}{|l|}{ Score: } \\
\hline Week 6 & $-10.0(7.6)$ & $-12.7(8.7)$ & $-11.8(7.9)$ & $-2.7(-5.44$ to -0.51$)$ & $-1.8(-4.43$ to 0.46$)$ & $-0.9(-2.67$ to 0.92$)$ \\
\hline Week 8 & $-12.0(8.4)$ & $-14.3(9.1)$ & $-13.8(8.5)$ & $-2.3(-4.97$ to 0.03$)$ & $-1.8(-4.50$ to 0.63$)$ & $-0.5(-2.22$ to 1.44$)$ \\
\hline \multicolumn{7}{|c|}{ Clinical global impression scale } \\
\hline \multicolumn{7}{|c|}{ Improvement (\%)‡: } \\
\hline Week 6 & 50.0 & 74.0 & 70.5 & 24 (13.1 to 39.0) & 20.5 (9.0 to 34.7$)$ & $3.5(-7.8$ to 14.7$)$ \\
\hline Week 8 & 65.3 & 77.0 & 74.2 & 11.7 (1.8 to 23.9$)$ & $8.9(-1.7$ to 20.1$)$ & $2.8(-7.5$ to 13.2$)$ \\
\hline \multicolumn{7}{|c|}{ Zung self rating depression scale } \\
\hline \multicolumn{7}{|l|}{ Score: } \\
\hline Week 6 & $-10.2(7.9)$ & $-14.1(11.3)$ & $-14.0(10.5)$ & $-3.9(-7.22$ to -1.59$)$ & $-3.8(-6.87$ to -0.62$)$ & $-0.1(-2.54$ to 2.38$)$ \\
\hline Week 8 & $-13.6(10.3)$ & $-17.4(13.6)$ & $-16.0(12.2)$ & $-3.8(-7.21$ to -0.75$)$ & $-2.4(-5.14$ to 1.35$)$ & $-1.4(-4.07$ to 1.34$)$ \\
\hline \multicolumn{7}{|l|}{ SF-36 } \\
\hline \multicolumn{7}{|c|}{ Physical component scale: } \\
\hline Week 8 & $4.7(6.7)$ & $8.2(10.9)$ & $5.8(9.9)$ & 4.5 (1.08 to 6.14$)$ & $1.1(-1.65$ to 3.67$)$ & $2.4(-0.02$ to 4.27$)$ \\
\hline \multicolumn{7}{|c|}{ Mental component scale: } \\
\hline Week 8 & $13.1(10.7)$ & $18.4(11.8)$ & $17.0(13.0)$ & 5.3 (1.92 to 8.70$)$ & $3.9(-0.24$ to 7.31$)$ & 1.4 (-1.68 to 4.12$)$ \\
\hline $\begin{array}{l}\text { Endpoint analyse } \\
{ }^{*} \text { For comparison } \\
\text { For Hamilton dep } \\
\text { differences, but i } \\
\text { †Patients with } \geqslant \\
\text { †Patients who in }\end{array}$ & $\begin{array}{l}\text { ast observati } \\
\text { treatment gr } \\
\text { d Hamilton a } \\
\text { clinical globs } \\
\text { t between ba } \\
\text { de "very muc }\end{array}$ & $\begin{array}{l}\text { rried forward } \\
95 \% \text { confide } \\
\text { scores and } f \\
\text { ression scale } \\
\text { and endpoint }\end{array}$ & $\begin{array}{l}\text { od for patients } \\
\text { ntervals for dif } \\
\text { ung self rating }\end{array}$ & $\begin{array}{l}0 \text { dropped out. } \\
\text { nces were calculated } \\
\text { ression scores, negat } \\
\text { ve values indicate bet }\end{array}$ & $\begin{array}{l}\text { "weighted for patients } p \\
\text { alues indicate better effica } \\
\text { ficacy of first treatment. }\end{array}$ & $\begin{array}{l}\text { centre" procedure. } \\
\text { of first treatment in }\end{array}$ \\
\hline
\end{tabular}

was a compromise between recommended mean dosage for efficacy ${ }^{13}$ and expected rate of adverse events. The participating doctors would not have accepted higher doses for the treatment of moderate depression owing to the expectation of frequent and severe side effects with consequences for compliance (increased drop out rates from the study) in this patient population. The 17 item version of the Hamilton depression scale has an inherent bias against tricyclics compared with both placebo and hypericum as it includes anticholinergic and other side effects in item 11 ("somatic anxiety"). Superiority of imipramine over placebo could be shown more clearly if this item was not included in the Hamilton depression total score (table 2). Whether this methodological bias or a suboptimal dosage of imipramine led to the less pronounced difference between drug and placebo in the Hamilton score cannot be answered conclusively. A recent meta-analysis of dose effect relations in 33 studies with imipramine ${ }^{14}$ showed no increased efficacy above 100-200 mg imipramine but a superior efficacy compared with doses below $100 \mathrm{mg}$. Although the rate of anticholinergic adverse events was in the range expected for tricyclics unblinding was not a major shortcoming in this study as only 39\% of imipramine treatments (hypericum extract 50\%, placebo 26\%) were correctly predicted by the investigators in this study in a retrospective assessment (data not shown).
Although modern antidepressants, such as selective serotonin reuptake inhibitors, are more suitable for comparison with hypericum because of their lower frequency of side effects, we chose imipramine mainly because tricyclics are still the most frequently prescribed antidepressants in primary care in Germany. This is because they cost less than modern antidepressants.

The rather high placebo response rate of $47 \%$ on the Hamilton depression scale after six weeks of treatment is within the known range for studies with hypericum extracts $(0 \% \text { to } 56 \%)^{4}$ and with synthetic antidepressants $(0 \%$ to $70 \%) .{ }^{15}$ Besides other factors (fluctuation of disease pattern, intense supervision

Table 3 Spontaneously reported or observed adverse events with total relative frequency $>3 \%$ and with probable or possible relation to trial drug and dropout rate due to adverse events (safety population). Values are numbers (percentages)

\begin{tabular}{lccc} 
& \multicolumn{3}{c}{ Group } \\
\cline { 2 - 4 } Variable & $\begin{array}{c}\text { Placebo } \\
(\mathbf{n}=\mathbf{4 7})\end{array}$ & $\begin{array}{c}\text { Hypericum extract } \\
(\mathbf{n}=\mathbf{1 0 6})\end{array}$ & $\begin{array}{c}\text { Imipramine } \\
(\mathbf{n}=\mathbf{1 1 0})\end{array}$ \\
\hline Patients with adverse events & $9(19)$ & $23(22)$ & $51(46)$ \\
\hline Dry mouth & $6(13)$ & $7(7)$ & $42(38)$ \\
\hline Nausea & $1(2)$ & $8(8)$ & $12(11)$ \\
\hline Constipation & $3(6)$ & $4(4)$ & $7(6)$ \\
\hline Headache & $1(2)$ & $3(3)$ & $6(6)$ \\
\hline Palpitation & 0 & $4(4)$ & $6(6)$ \\
\hline Dizziness & $1(2)$ & $1(1)$ & $7(6)$ \\
\hline Dropout rate due to adverse events & 0 & 0 & $1(1)$ \\
\hline
\end{tabular}


according to the study setting), it may be either a result of a possible selection process of patients with a good prognosis in placebo controlled trials of hypericum or methodologically biased by the smaller size of the placebo group, which resulted in a maximum of four patients compared with 20 on active drugs per centre.

\section{Quality of life and aspects of tolerability}

Quality of life ratings with SF-36 were not included in clinical trials with hypericum extract and, with one exception, ${ }^{16}$ were not sensitive for differences between drug and placebo. We found differences to be largest in the mental component scale, but only hypericum extract was superior to placebo in the physical component scale. The higher rate of adverse events in the imipramine group is probably responsible for a lesser improvement in the physical component compared with the mental component

Tolerability analysis showed a higher rate of adverse events in the imipramine group than in the other two treatment groups, which was primarily due to dry mouth. Generally, the observed favourable safety profile of hypericum extract agrees with the data from a recently published review. ${ }^{17}$

\section{Conclusions}

Since hypericum products may vary considerably in composition (total hypericins, hyperforin, flavonol derivatives) the results cannot be generalised to other extracts. Furthermore, the tested daily dosage of 1050 mg extract, which is equivalent to $6 \mathrm{~g}$ of the crude herb, is higher than that recommended and mostly used for the treatment of mild depression. In the first three arm comparative trial of hypericum extract, we showed that at an average dose of $350 \mathrm{mg}$ three times daily hypericum extract STEI 300 was a more effective antidepressant than placebo and at least equally effective to 100 $\mathrm{mg}$ imipramine daily in the treatment of moderate depression. Also, any side effects with hypericum extract did not impair important quality of life measures. Since many depressed patients receive either no treatment or inadequate treatment after an initial depressive episode and are at increased risk of recurrence of such episodes, ${ }^{18}$ hypericum extract may thus be considered as an alternative first choice treatment in most cases of mild to moderate depression without psychotic symptoms.

We thank the doctors who contributed to the study: $\mathrm{H}$ Carboni, E Homsy, P Sandow, E Schwittay, H Wiswedel, D Breitfelder, M Oelker, A Berger, K D Bergert, H Hauer, A Naumburger, E Mandrella, R Krone, W Englisch, P Unterberg, H Leykauf, J Blasy, and M Staudinger.

Contributors: MP, RK, and KOH developed the study protocol and are guarantors for the paper. RK and $\mathrm{KOH}$ wrote the first draft of the manuscript, which was discussed, revised, and accepted by all authors. RK and his team at IMEREM analysed the data and provided the integrated study report. MP, as the principal investigator of the trial, was primarily responsible for the clinical interpretation of the results.

Funding: Steiner Arzneimittel, Berlin, Germany.

Competing interests: $\mathrm{KOH}$ is an employee of Steiner Arzneimittel. RK is head of a contract research organisation (IMEREM), which is engaged in several clinical trials with hypericum extract for different pharmaceutical companies.

1 Baldessarini RJ. Drugs and the treatment of psychiatric disorders: depression and mania. In: Hardman JG, Limbird LE, Molinoff PB, Ruddon RW, Gilman AG, eds. Goodman and Gilman's the pharmacological basis of therapeutics, 9th ed. New York: McGraw-Hill, 1996:431-59.
Key messages

- Hypericum extract (STEI 300) was effective after 4,6 , and 8 weeks of treatment in patients with moderate depression

- Simultaneous analysis confirmed hypericum extract to be at least as efficacious as imipramine $100 \mathrm{mg}$ daily after eight weeks of treatment

- Besides better antidepressive efficacy both hypericum extract and imipramine improved quality of life

- Patients tolerate hypericum extracts much better than they do tricyclics and therefore by improving patients' compliance hypericum extracts are promising drugs for long term treatment

2 Lépine JP, Gastpar M, Mendlewicz J, Tylee A on behalf of the Depression Research in European Society Steering Committee. Depression in the community: the first pan-European study DEPRES (depression research in European society). Int Clin Psychopharmacol 1997;12:19-29.

3 Linden M, Maier W, Achberger M, Herr R, Helmchen H, Benkert O. Psychiatric diseases and their treatment in general practice in Germany. Results of a World Health Organization (WHO) study. Nervenarzt 1996;67:205-15

4 Linde K, Mulrow CD. St John's wort for depression. In: Cochrane Collaboration. Cochrane Library. Issue 2. Oxford: Update Software, 1999.

5 De Smet PAGM, Nolen WA. St John's wort as an antidepressant. BMJ 1996; 313:241-2.

6 Volz HP. Controlled clinical trials of hypericum extracts in depressed patients-an overview. Pharmacopsychiat 1997;30(suppl):72-6.

7 The European Commission. Adapted guideline from ICH-E9: note for guidance on statistical priniciples for clinical trials. London: European Agenc for the Evaluation of Medicinal Products, 1998. [CPMP/ICH/363/96.]

8 The European Commission. Adapted guideline from ICH-E6: note for guidance on good clinical practice. London: European Agency for the Evaluation of Medicinal Products, 1997. [CPMP/ICH/135/95.]

9 The European Commission. Note for guidance: medicinal products for the treatment of depression. Brussels: Committee for Proprietary Medicinal Products, 1990

10 Lehmacher W. Äquivalenznachweise bei Multicenter-Studien. In: Michaelis J, Hommel G, Wellek S, eds. Europäische Perspektiven der Medizinischen Informatik, Biometrie und Epidemiologie. Munich: MMV Medizin Verlag, 1993:152-4.

11 Montgomery SA. Clinically relevant effect sizes in depression. Eur Neuropsychopharmacol 1994;4:283-4.

12 Röhmel J. Therapeutic equivalence investigations: statistical considerations. Statist Med 1998;17:1703-14.

13 German Commission A. Monograph imipramine. Bundesanzeiger 1992, Aug 8.

14 Bollini P, Pampallona S, Tibaldi G, Kupelnick B, Munizza C. Effectiveness of antidepressants: meta-analysis of dose-effect relationships in randomised clinical trials. Br J Psychiat 1999;174:297-303.

15 Preskorn SH, Burke M. Somatic therapy for major depressive disorder: selection of an antidepressant. J Clin Psychiatry 1992;53(suppl):5-18

16 Heiligenstein JH, Ware JE Jr, Beusterien KM, Roback PJ, Andrejasich C, Tollefson GD. Acute effects of fluoxetine versus placebo on functional health and well-being in late-life depression. Int Psychogeriatr 1995; (suppl):125-37.

17 Ernst E, Rand JI, Barnes J, Stevinson C. Adverse effects profile of the herbal antidepressant St John's wort. Eur J Clin Pharmacol 1998;54:589-94.

18 Angst J. A regular review of the long-term follow-up of depression. BMJ 1997;315:1143-6.

(Accepted 31 August 1999)

\section{Endpiece \\ On ageing: the optimist}

Is not old wine wholesomest, old pippins toothsomest, old wood burn brightest, old linen wash whitest? Old soldiers, sweetheart, are surest, and old lovers are soundest.

Westward Hoe, John Webster, c 1580-1625

Submitted by Fred Charatan, retired geriatric psychiatrist, Florida 


\title{
Commentary: Has hypericum found its place in antidepressant treatment?
}

\author{
Klaus Linde, Michael Berner
}

Hypericum extracts are the most widely prescribed antidepressants in Germany and their popularity in other countries is increasing. The study of Philipp et al as well as other new trials ${ }^{12}$ confirm the existing evidence ${ }^{3}$ that such extracts are more effective than placebo in mild and moderately severe depression. The most relevant questions for clinical research are now whether hypericum is really as effective as standard antidepressants, whether it is effective and safe for long term treatment and for more severe forms of depression, and how different extracts and dosages compare.

The trial of Philipp et al is a relevant contribution to the first of these questions but also highlights some of the common problems in research on hypericum extracts. Existing trials have been criticised for comparing hypericum with low doses of standard antidepressants. This criticism also applies to some extent to this trial. The authors argue that higher doses would not have been accepted by the participating practitioners owing to the high rate of expected side effects. The comparison with the placebo control group shows that both treatments had specific effects. Nevertheless, the conclusion from this trial can only be that a comparatively high dose of the tested hypericum extract seems to be similarly effective as a comparatively low dose of imipramine.

The patients receiving the hypericum extract or placebo experienced less and different side effects than those taking imipramine. This might have caused some unblinding. It cannot be ruled out that this had some influence on the comparisons of imipramine versus placebo and imipramine versus hypericum. Unblinding is a possibly common but mostly undiscussed issue in many clinical studies. In our opinion, testing for unblinding should become routine in clinical trials. Philipp et al conclude from a retrospective assessment that unblinding was not a major shortcoming in their study, but without a more detailed description of how exactly this assessment was performed this statement cannot be substantiated by the reader. For a long time imipramine has been the gold standard for comparison in clinical trials of antidepressant treatment
Therefore, a comparison with imipramine is legitimate. But it would be important to know how hypericum extracts compare with selective serotonin reuptake inhibitors for both efficacy and side effects. So far there is only one trial comparing another hypericum extract with (the comparatively low dose of $20 \mathrm{mg}$ ) fluoxetine, ${ }^{4}$ which found similar effectiveness and rates of side effects in patients with mild to moderately severe depression. A large trial sponsored by the US National Center for Complementary and Alternative Medicine comparing hypericum, a selective serotonin reuptake inhibitor, and placebo in patients with major depression is ongoing.

The superiority of hypericum extract and imipramine over placebo is not very impressive in the trial by Philipp et al. However, the patients in the placebo group experienced considerable improvement, which seems plausible in moderately depressed patients in primary care. An interesting finding of the trial is also that hypericum extract seemed to have had a significant effect on quality of life.

In summary, this trial adds to the growing evidence on the effectiveness of hypericum in mildly and moderately depressed patients. Still, our personal differences on which treatment we would prefer in case we had a moderately severe depression indicates that the place of hypericum in antidepressant treatment is not yet fully established. Whereas one of us would prescribe a chemically defined antidepressant with a low side effect profile the other would first try hypericum.

Competing interests: $\mathrm{MB}$ has received sponsorship from Schwabe in the past, manufacturer of a hypericum extract.

1 Schrader E, Meier B, Brattström A. Hypericum treatment of mild-moderate depression in a placebo-controlled study. A prospective, double-blind, randomized, placebo-controlled, multicentre study. Hum Psychopharmacol 1998;13:163-9.

2 Laakmann G, Dienel A, Kieser M. Clinical significance of hyperforin for the efficacy of hypericum extracts on depressive disorders of different severities. Phytomedicine 1998-5:435-42.

3 Linde K, Mulrow CD. St John's wort for depression. In Cochrane Collaboration. Cochrane Library. Issue 1. Oxford: Update Software, 1999.

4 Harrer G, Schmidt U, Kuhn U, Biller A. Comparison of equivalence between St John's wort extract LoHyp-57 and fluoxetine. Arzneim-Forsch Drug Res 1999;49:289-96.
Centre for

Complementary Medicine Research, Department of Internal Medicine

II, Technische

Universität

München, Munich,

Germany

Klaus Linde

senior researcher

Department of Psychiatry and Psychotherapy,

Albert-LudwigsUniversität, 79104 Freiburg, Germany Michael Berner clinical psychiatrist

Correspondence to: K Linde

Klaus.Linde@lrz. tu-muenchen.de

\section{Email submissions from outside the United Kingdom}

We are now offering an email submission service for authors from outside the UK. The address is papers@bmj.com

Ideally our email server would link seamlessly with our manuscript tracking system, but for now it does not, which is why we are offering the service only to authors outside the UK. Most post in the UK arrives the next day, so UK authors have the least to gain in speed of delivery from email delivery. As soon as our systems improve we will invite email submissions from everyone.

If you choose to send your submission by email please would you send the text and any tables and figures as attached files, together with a covering letter giving all your contact details (postal address, phone, fax, and email address). We can read files created with most word processing, graphics, and spreadsheet programs.

When your submission is received in our email box you will receive an automatic acknowledgment to show that it has arrived. If the submission is incomplete we will contact you and ask you to resend the missing information.

Once the submission is complete we will register it on our manuscript tracking system and you will receive a standard acknowledgment in the post.

Letters to the editor should continue to be sent direct to www.bmj.com as rapid responses or to letters@bmj.com 